\title{
Deciphering the secret code: A new methodology for the cross-cultural analysis of nonverbal behavior
}

\author{
Gary Bente, Maria Senokozuieva, and Sibylle Pennig \\ University of Cologne, Cologne, Germany \\ AHMAD AL-ISSA \\ American University of Sharjah, Sharjah, United Arab Emirates \\ AND \\ OLIVER FISCHER \\ Bertelsmann AG, Gütersloh, Germany
}

\begin{abstract}
This article addresses methodological issues in the description and evaluation of nonverbal behavior (NVB) in cross-cultural studies. Videos containing cultural samples of NVB are of little use when analyzing the effects of NVB across cultures. Since video recordings confuse visible behavior with the physical appearances of the actors, they are likely to activate stereotypes derived from hints about an actor's culture and ethnicity that override the effects of the observed NVB. As a solution for this problem, we suggest here a unified computerized method for the transcription and experimental simulation of NVB. This approach makes use of advanced 3-D animation tools to generate detailed protocols of NVB that can be used to generate culture-free stimulus materials using standardized virtual characters.
\end{abstract}

In today's globalized world, the ability to communicate and collaborate across national and cultural borders is increasingly important (Lee, Matsumoto, Kobayashi, Krupp, \& Maniatis, 1992). The need for reciprocal understanding grows not only at the political and institutional levels, but also - and maybe even more importantly-at the level of individual interpersonal encounters. Even when there is no direct contact, a permanent supply of behaviors and social practices from other countries is provided by the mass media-for example, through TV news coverage, documentaries, or even fictional offerings. These images are often the only source of information about other cultures, so it is to be expected that they not only influence our impressions of the portrayed individuals but also shape our attitudes toward the cultures of their origin.

Research has shown that the way we form our impressions of other people is far more dependent on the nonverbal behavior (NVB) we observe than on the spoken words we listen to (Argyle, Salter, Nicholson, Williams, \& Burgess, 1970; Burgoon, 1994; Mehrabian \& Ferris, 1967; Mehrabian \& Wiener, 1967; Newcombe \& Ashkanasy, 2002). In most instances, nonverbal cues are processed more quickly than verbal messages, and therefore are usually responsible for our first impressions. First impressions/ judgments are often based on facial expressions, postures, gestures, use of personal space and distance, general ap- pearance and attire, eye contact and gaze, and so on. Although much of our nonverbal communication is "part of a universally recognized and understood code" (Burgoon, Buller, \& Woodall, 1996, p. 5), a great deal is rooted in our culture-and can therefore be culture-specific. This fact is of particular importance in terms of the perception of people from other cultures and the outcomes of intercultural communication, because the standards we apply and the judgments we make are in turn subject to culture-specific interpretations. Matsumoto (2006) concluded that "collectively, the evidence provides more than ample support for the notion that culture plays a large role in molding all of our nonverbal behaviors, which comprise an important part of the communication process" (p. 221). Cultures can differ remarkably with respect to social values and the nature of interpersonal relationships (Hofstede, 1980). These differences are particularly subtle in interpersonal communication and NVB (Kowner \& Wiseman, 2003), but the effects can be as substantial as communication breakdowns, ambivalence, or conflict.

In contrast to verbal communication, the central elements of nonverbal communication and their meanings cannot be looked up in a dictionary. Nonverbal signal production, to a large degree, is not rule-based, nor does it follow an explicit grammar. This fact not only complicates cross-cultural communication in practice, but also poses 
specific methodological problems for the researcher. Cultural differences in NVB are very difficult to describe objectively, and the experimental manipulation of specific nonverbal cues is usually confounded by nuisance variables. The present article focuses on these methodological problems and introduces a unified, computer-based approach to their solution. In the first section, we will address basic mechanisms of nonverbal communication in relation to culture and point out methodological problems inherent in their analysis. Second, we will address the possibilities of nonbiased description and evaluation of nonverbal behaviors across cultures and suggest a sequential research strategy using state-of-the-art computer animation technology. On this basis, we will introduce a software platform for the transcription and experimental simulation of movement behavior and demonstrate its unique possibilities by referring to a recently launched cross-cultural study in Germany, the United States, and the United Arab Emirates.

\section{Culture and Nonverbal Communication}

Beginning with Efron's pioneering work on gestures (Boas \& Efron, 1936; Efron, 1941), a considerable number of studies have demonstrated the cultural variety in the production and interpretation of various NVBs, such as facial expressions (Ekman, 1972; Ekman et al., 1987), emblematic gestures (Ekman, 1976; Friesen, Ekman, \& Wallbott, 1979), body postures (Kudoh \& Matsumoto, 1985; Matsumoto \& Kudoh, 1987), interpersonal distance (Hall, 1976), and eye contact (Hall \& Hall, 1990; Kitao \& Kitao, 1989). The common thread in all of these approaches is that they address well-described spatiotemporal activity patterns that can easily be isolated from the behavioral stream, or even directly measured, and for which specific functional a priori hypotheses can be formulated. Beyond such "meaningful" units, however, more subtle nonverbal signals can be observed in interpersonal communication, signals that bypass conscious attention and registration but nevertheless have a strong influence on our interpersonal impressions and social relationships. Recent research from human ethology and cognitive neuroscience clearly shows that movement qualities, such as speed, acceleration, and dimensional complexity can carry relevant social information (Grammer, Honda, Juette, \& Schmitt, 1999; Krumhuber \& Kappas, 2005). The authors of these studies have pointed out that the dynamic qualities of NVB can have an even stronger impact on the observer's impressions than so-called semantic aspects, although they might not be consciously processed or identified as a possible cause. It is evident that semantic and nonsemantic aspects of NVB are interwoven in real-life interactions and can hardly be separated by the observer, neither with respect to their physical properties nor with respect to their particular socioemotional effects. Actual conceptions hold that nonverbal communication can be considered as analogous, widely automated, and unconscious in production and in reception (Buck, Losow, Murphy, \& Costanzo, 1992; DePaulo \& Friedman, 1998).
The subliminal nature of nonverbal communication is also underlined by the facts that impressions based on nonverbal cues are formed within extremely short time periods (Dimberg, 1997) and that even very subtle nonverbal variations can have a significant influence on the observer's perception of the sender (Frey, 1999; Frey, Hirsbrunner, Florin, Daw, \& Crawford, 1983). The growing knowledge about the "nature" of nonverbal communication has forced researchers to broaden their analytical perspective to capture variations beyond predefined signals, and at the same time to refine the resolution of observation in order to account for more subtle and transient variations.

Although these implications concern the descriptive analysis of NVB, cultural comparisons face further methodological problems when it comes to the investigation of nonverbal communication effects. We know that the evaluation of another person and the perception and interpretation of his or her behavior is often biased by prior categorical judgments, such as stereotypes or even prejudice. For example, white subjects judge the behavior of a black person to be more violent than the same behavior exhibited by a white person (Duncan, 1976). Moreover, not only person perception is affected by this categorization based on culture or race, but also the interaction behavior toward, for instance, a black person. Thus, Dovidio, Kawakami, and Gaertner (2002) showed that skin color has a crucial impact on verbal as well as nonverbal communication patterns of interlocutors. This influence is often unconscious and is exerted by even the most subtle stimulations. For example, Chen and Bargh (1997) showed that subliminal presentations of black faces caused more hostile behavior in white participants than did similar presentations of white faces. From these results, it can be expected that photo or video samples from other cultures more or less automatically activate stereotypes as they convey category-relevant information tied to physical appearance. This fact can cause severe confusion in the analysis of impression effects, making it difficult to separate category-based, top-down processes and behavior-based, bottom-up processes in person perception. These methodological problems are not specific to the analysis of cultural differences, but are instead inherent in the analysis of nonverbal communication in general. It must be stated, however, that conceptual and instrumental advances in other areas of nonverbal communication research have not yet been systematically adapted to cross-cultural research, although the possibility of progress, as we hope to show, is in many respects self-evident.

\section{Description and Evaluation of NVB: Methodological Considerations}

A look at the relevant research literature reveals two types of nonverbal communication studies. The first type focuses on the production side (i.e., on similarities and differences in overt NVB), whereas the second type focuses on the reception side (i.e., on similarities and differences in the sensitivity to and the interpretation of nonverbal cues). With respect to the analysis of cultural differences, 


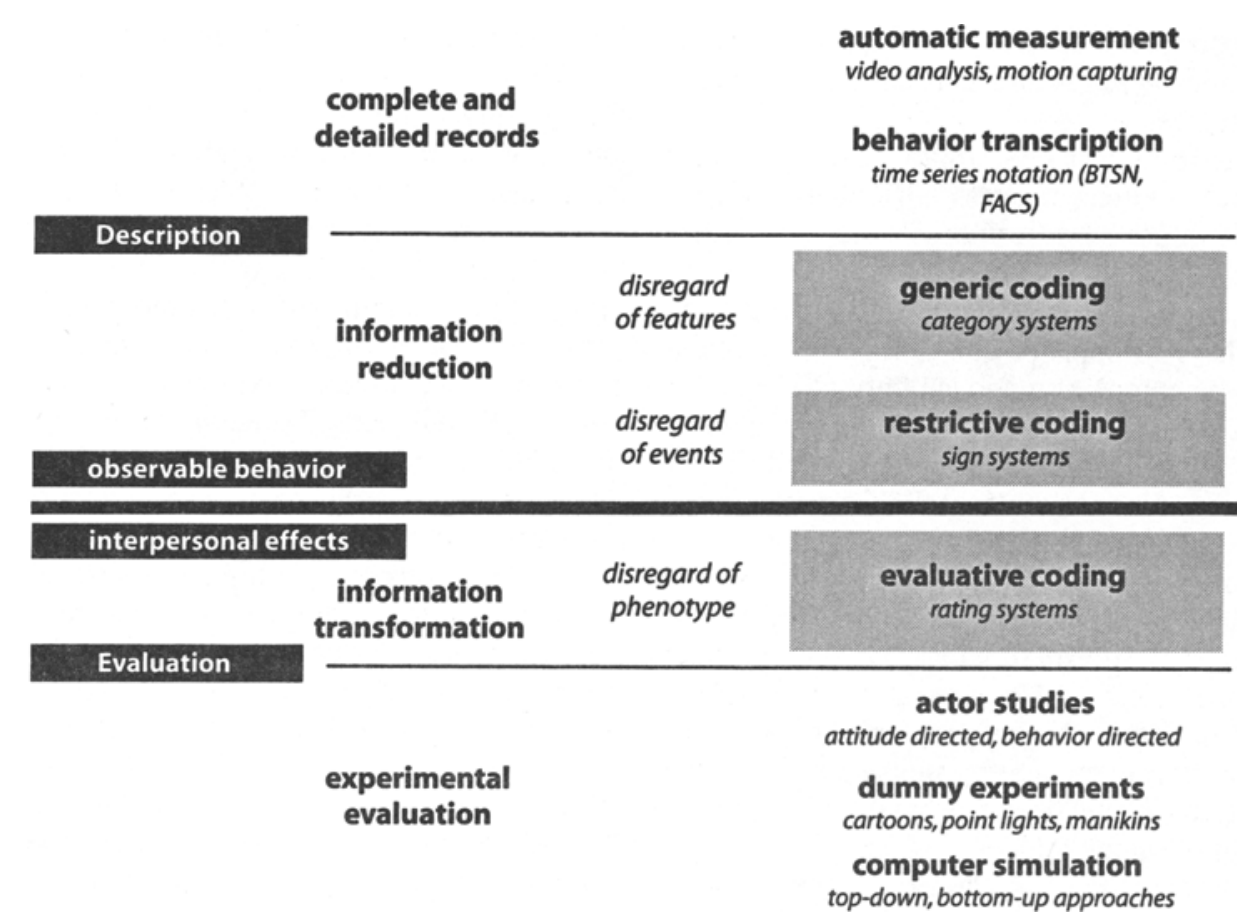

Figure 1. Separation of descriptive and evaluative tasks in nonverbal communication research (for a detailed explanation, see the text).

both aspects are interwoven. Identical nonverbal patterns can have different meanings and create distinct effects in different cultures, whereas the same socioemotional consequences can arise from different NVBs. For the scientific investigator, this functional commingling poses a specific methodological problem that can only be addressed by means of istrict separation between descriptive and evaluative aspects of the research task. This means that the collection of behavioral data must by no means be influenced by cultural and personal assumptions about the relevance and adequacy of particular nonverbal cues. In addition, it must be ensured that the comparative evaluation - that is, the analysis of the effects of culturally diverse behaviors-is not influenced by specific expectations or stereotypes on the part of the perceiver. However, in a critical methodological review, Frey and Pool (1976) posited that traditional nonverbal communication research does not address the strict separation mentioned above. Figure 1 illustrates the core aspects of this early critique and points to specific solutions to this dilemma that have recently been made possible by modern computer technology.

According to Frey and Pool (1976), traditional research in NVB has referred to one of three coding principles, all of which imply information reduction strategies based on implicit assumptions or explicit hypotheses (see Figure 1). (1) Generic coding aims at exhaustive descriptions of observed behavior using rough categories (e.g., an upright body posture) while ignoring behavioral details (e.g., dynamic properties of body movements). Generic coding is mostly explorative in nature. (2) Restrictive coding aims at a most accurate registration and quantification of particular nonverbal cues that serve an explicit communication function (e.g., an emblematic hand gesture), and it is mainly used to test specific hypotheses. Both generic and restrictive coding procedures rely on the subjective assessments of coders. However, as stated above, social meaning can also be conveyed by subtle cues or by general physical properties of movements, which do not pass the threshold of conscious registration. In those cases, both coding strategies (generic and restrictive) will miss important parts of the behavioral variance because they rely on accurate signal detection on the part of human coders. (3) Evaluative coding, in contrast to generic and restrictive coding, directly transforms the observed behavior into psychological categories (e.g., scoring the aggressiveness or friendliness of a behavioral sequence). These data protocols consist not of behavioral descriptions, but of the subjective impressions of observers. Such methods can be highly useful for the analysis of the effects of nonverbal communication, although small numbers of coders should be then replaced by random samples of observers from the relevant target groups (e.g., different cultures). Although evaluative ratings have been used repeatedly to identify behavioral differences, it should be pointed out that evaluative coding does not provide descriptions of behavior, but rather assessments of the applicability of certain psychological categories. Instead of the criterion of objectivity, the criterion of intersubjectivity is substituted - that is, the quality of measurement is quantified via interrater reliability (i.e., correlation of the judgments). This does not mean that evaluative coding procedures generally yield 
invalid data, only that evaluative coding is inadequate as a means of behavior description and, consequently, as a tool for production studies.

The distinction between descriptive and evaluative phase in the measurement process is fundamental. As a first step, NVB must be described objectively in different cultures, and as a second step, its interpersonal effects must be tested in different cultures independently. As a result, we have to improve our methods in both directions. Given the complexity and subtlety of NVB, the major problem in the descriptive task is the availability of standardized procedures for an unbiased, detailed, and exhaustive notation of human motions. Performance in the evaluative research task will depend on a researcher's ability to experimentally control specific behavioral aspects and to isolate the influence of behavioral cues from that of other socially relevant information that is tied to physical appearance (e.g., ethnicity, color of the skin, clothing).

As indicated in Figure 1, the problems of behavior description have been overcome by physicalistic coding systems - for example, the "Bernese system for time series notation of movement behavior" (BTSN; Frey et al., 1983; Frey, Hirsbrunner, Pool, \& Daw, 1981; Frey \& Pool, 1976) or the "facial action coding system" (FACS; Ekman $\&$ Friesen, 1978). Although they still require human coders, these systems make use of detailed behavior protocols that enable high degrees of reliability and objectivity. In contrast to the term "coding," we suggest the term "transcription" for this type of data collection, because the underlying notation principles are equivalent to those in speech notation. Just as speech can be noted as a series of sound symbols, movement can be codified as a sequence of positional states of various body parts (see Donaghy, 1989; Frey et al., 1983). The "nonverbal alphabet" required for this task is certainly more complex than the verbal one. Each body part can independently vary in six spatial dimensions (translation along the axes plus rotation around three axes), and within these dimensions, we have to provide a sufficient number of codes to account for all movement variations that can be detected in a video recording. Detailed descriptions of the BTSN coding principles can be found in Frey et al. (1983; see also Bente, 1989, 2002).

Transcription methods such as the BTSN are time consuming and expensive. Depending on the amount of movement activity in a given time unit, the time series notation can take up to 200 times as long as the duration of the analyzed video sequence. Furthermore, BTSN is performed on an ordinal scale level, neglecting the precise angular degrees and spatial distances involved in the rotation and translation of the body parts. In order to use these data protocols for computer animations, additional fine tuning is necessary, which again can take 100 times the length of the video. Economic and more precise alternatives to such transcription procedures are provided in the form of motion capture devices, such as position trackers and data gloves, that allow for the direct measurement of movement behavior (see Bente \& Krämer, 2004). It must be noted, however, that these devices are quite obtrusive and often impractical for use in natural settings, since they require the attachment of sensors and the installation of stationary laboratory equipment. Recent developments have made use of state-of-the-art computer animation software to facilitate the notation process rather than replacing it with direct measurement. Interfacing with 3-D rendering engines, these computer-aided notation tools issue data formats compatible with those from motion capture devices containing synchronized vectors of translations and rotations of all body parts. The temporal and spatial resolution of such time series protocols, as well as their accuracy, is very high, and they can be used as direct inputs for realistic computer animations of human behavior.

The direct link to 3-D animation also offers a solution to the second research problem mentioned above: the experimental study of person perception effects induced by NVB (see Figure 1). Actor studies that use confederates to carry out specific behaviors have repeatedly failed, because actors find it impossible to vary singular aspects of NVB while keeping all other variables constant (Lewis, Derlega, Shankar, Cochard, \& Finkel, 1997). Moreover, actor studies still imply the visibility of personal characteristics (physical appearance) that might evoke stereotypes. Dummy studies using photographed postures of wooden manikins, on the other hand, allow for the control of physical appearance but miss out on dynamic aspects of NVB. Computer simulations of NVB based on time series protocols offer a unique possibility to solve both of the problems of traditional effect studies. On one hand, they allow for dynamic presentations of NVB (realistic 3-D animations), and on the other, they offer the possibility of masking or changing aspects of physical appearance that might be indicative of the cultural background of the original actor. The general usefulness and validity of computer simulation techniques in NVB research has been amply demonstrated in previous studies, showing that 3-D animations of NVB lead to the same impressions as the original video sequences they were based on. Strikingly, this finding was true even though the animation did not include facial expressions (Bente, Krämer, \& Petersen, 2002; Bente, Krämer, Petersen, \& de Ruiter, 2001; Frey, 1999).

In the following section, we will introduce a novel computerized approach to the transcription and experimental simulation of NVB, focusing on movement behavior; this new tool has been developed with special emphasis on its use in cultural comparison studies.

\section{A Unified Platform for the Transcription and Simulation of NVB}

The method described in this section was developed within a cross-cultural research project that focuses on power-related NVBs in Germany, the United States, and the United Arab Emirates, as occurring in mass media (self-presentation of politicians in the news) as well as in dyadic managerial interactions. The project was sponsored by the German Science Foundation as part of a 
12-year Special Interest Program in "Media and Cultural Communication" at the University of Cologne. Since the present article focuses on methodological rather then theoretical details, the cross-cultural study will only be used to exemplify the power of the novel research tools, and the specifics of the study will not be reported in detail here.

The introduced software platform builds on developments laid out in the late 1980s (Bente, 1989). Whereas traditional time series coding used paper and pencil to note down video movement on a frame-by-frame basis, subsequent software developments were aimed at facilitating the transcription process by integrating data protocols and digital video into one computerized coding environment. Our early approaches aimed at incorporating 3-D animation modules of human bodies, controlled by the time series data, which gave us direct visual feedback on the coding quality (Bente, 1989; Bente, D'Alessandro, Fürtjes, \& Krämer, 1997) and also enabled an offline experimental animation of completed protocols (Bente, Feist, \& Elder, 1996; Bente et al., 2002; Bente, Krämer, Petersen, \& de Ruiter, 2001; Bente, Krämer, Trogemann, Piesk, \& Fischer, 2001). The main restrictions of these methods related to ease of use, the real-time capacity of the animation tools, the realism of the human body models, the exchangeability and editing of the models, and the export of movement data for analytical purposes. Current developments therefore make use of state-of-the-art computer animation software and realize the scientific requirements as plug-in modules.

The new system is based on a commercial character animation tool (Alias Motion Builder 7.5), running under Windows XP. A standard PC with $1.8 \mathrm{GHz}$ or more and an Open GL graphics board is sufficient to run the application. Alias Motion Builder has to be purchased separately; further information and system requirements about Motion Builder can be found at usa.autodesk.com/ adsk/servlet $/$ index?siteID $=123112 \&$ id $=6837879$. For the specific purposes of this project, a special movement transcription plug-in for Motion Builder was developed, including a synchronized data spreadsheet, a video playback window, and a real-time animation window. The emphasis in the development was on usability for communication researchers. The main characteristics include a convenient way to code movement, easy data access, flexible data import and export, and accurate real-time animation.

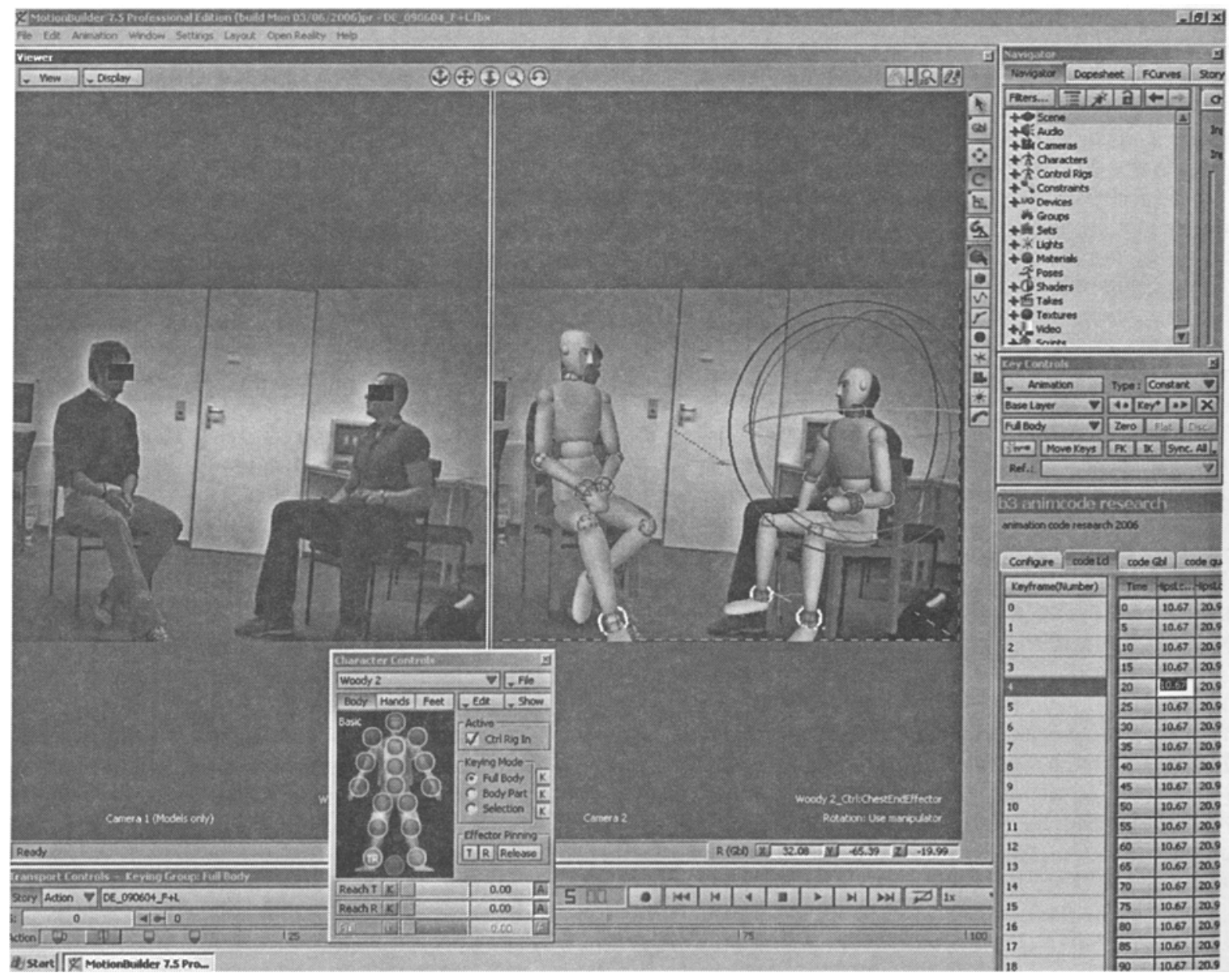

Figure 2. Screen shot showing the user interface of the coding and simulation platform (for a description, see the text). 
Figure 2 shows a snapshot of the user interface of the transcription and animation platform. As is visible in this figure, the interface provides two display windows, showing the original video (left) and the related computer animation (right) of two 3-D characters. The video can also be switched on in the second window to allow for a direct superimposition of the video with the 3-D model. In this case, the 3-D character can also be displayed as a transparent wire frame model, thus enabling a direct check of the code quality by comparing the body postures of the original actors and the adjusted postures of the computer models. Both display windows are time synchronizedthat is, advancing one frame in the left window will cause advancement in the right, and vice versa. Furthermore, in "play" mode, both windows show the dynamic activity of the originals and the models in real-time. The animation window is synchronized with the movement protocol, which is also displayed as a spreadsheet at the right side of the interface. Changes in the spreadsheet also cause corresponding changes in the models' postures. The columns in the spreadsheet represent the coding dimensions - that is, the six spatial degrees of freedom (XYZ rotation and $\mathrm{XYZ}$ translation) for each body part (head, arms, hands, trunk, etc.). The lines hold the temporal information, with an interval of five key-frames per second (five lines contain $1 \mathrm{sec}$ of movement).

The coding interval can be freely chosen. A minimum resolution of two key-frames per second, however, is recommended in order to achieve a realistic reproduction of the original behavior. When generating computer animations, the key-frame data are interpolated to a frame rate of 25 or 30 frames per second, using Bezier interpolation to guarantee the smooth flow of movements. The graphical user interface allows for different coding modalities following either the principles of either forward or inverse kinematics. In forward kinematics mode, the coder influences the local rotation angles of a specific body part, which also leads to a translation of the body part attached to the next joint-for example, changing the rotation angle of the lower arm in the elbow articulation will lead to a translation of the hand. In inverse kinematics mode, the coder influences the translation (spatial position) of a body part (e.g., the hand), leaving it to the system to establish the most likely rotations in the superordinated joints. The coder is free to choose the modality in each instance for his/her convenience. For example, it is easier to adjust the position and rotation of the upper extremities by translation of the hands (inverse kinematics), whereas the upper body and the head are much easier to adjust by using local rotation angles (forward kinematics). It must be posited, however, that regardless of the chosen coding modality, the data matrix always contains the full set of rotation as well as translation data for all body parts and joints. This is another clear advantage of our approach over traditional BTSN coding, in which translation and rotation data are exclusive for certain body parts and are mixed in the final data matrix.

Technically, the coding is done via direct mouse dragging of selected body parts (inverse kinematics) or via a
3-D sphere depicted in the animation window (see Figure 2) that is used to adjust the local rotation angles of joints (forward kinematics). The various body parts can be selected by a direct mouse click onto the character or via a small selection window at the bottom of the screen. When performing an adjustment, all affected angles and positions (rotations and translations) are updated in the internal data matrix and transferred into the spreadsheet. Both translation and rotation data are thus accessible for later analysis of postures, movements, and orientations. The richness of information provided by these movement protocols justifies the still considerable time and effort that has to be invested in the coding procedure. At a level of resolution comparable to traditional BTSN coding, a 1-min video recording may require up to $150 \mathrm{~min}$ of coding time. However, in contrast to the BTSN, the resulting data do not need any additional fine tuning and can be used directly for experimental computer animations. The new methodology thus takes about half the coding time of traditional BTSN and at the same time offers much higher accuracy. Because the program interface is most intuitive, coder training can be completed within 2 days. The performance curve for the coders is asymptotic, reaching maximum levels of certainty and speed after about 1 week. After completion of the transcription process, 3-D rendering of the coded movement sequence can be launched. Specific output options of the program allow for the selection of frame rate, interpolation type, and video compression.

The highly accurate and editable behavior protocols resulting from the computer-aided transcription provide a unique basis for cross-cultural production as well as reception studies of NVB. In particular, the novel methodology facilitates three research strategies: (1) structural analysis and cross-cultural comparisons of observed NVB, (2) culture-fair person perception studies based on computer-animated characters, and (3) person perception studies based on variations of specific nonverbal cues.

For the purpose of comparative structural analysis of NVB, data protocols are exported as tab-delimited ASCII files, which can be read by Excel, SPSS, or comparable data management or statistics software. Special tools for graphical and quantitative analysis of these data sets have been provided, allowing for the extraction of specific behavioral dimensions-for example, head or body orientation toward the interlocutor-as well as for the aggregation and integration of different dimensions-for example, when calculating the overall motion complexity (number of dimensions involved in any movement) or the symmetry of body postures within and between the interlocutors. Strategies for explorative and quantitative analyses of multiple time series protocols of NVB, as well as various parameters, have been described in previous work (see Bente, Donaghy, \& Suwelack, 1998; Frey et al., 1983; Suwelack \& Bente, 1995).

With respect to the reception of NVB, the methodology enables the use of these movement protocols for culturefair person perception experiments. As a basis for judgment studies, the software issues very realistic, smooth 


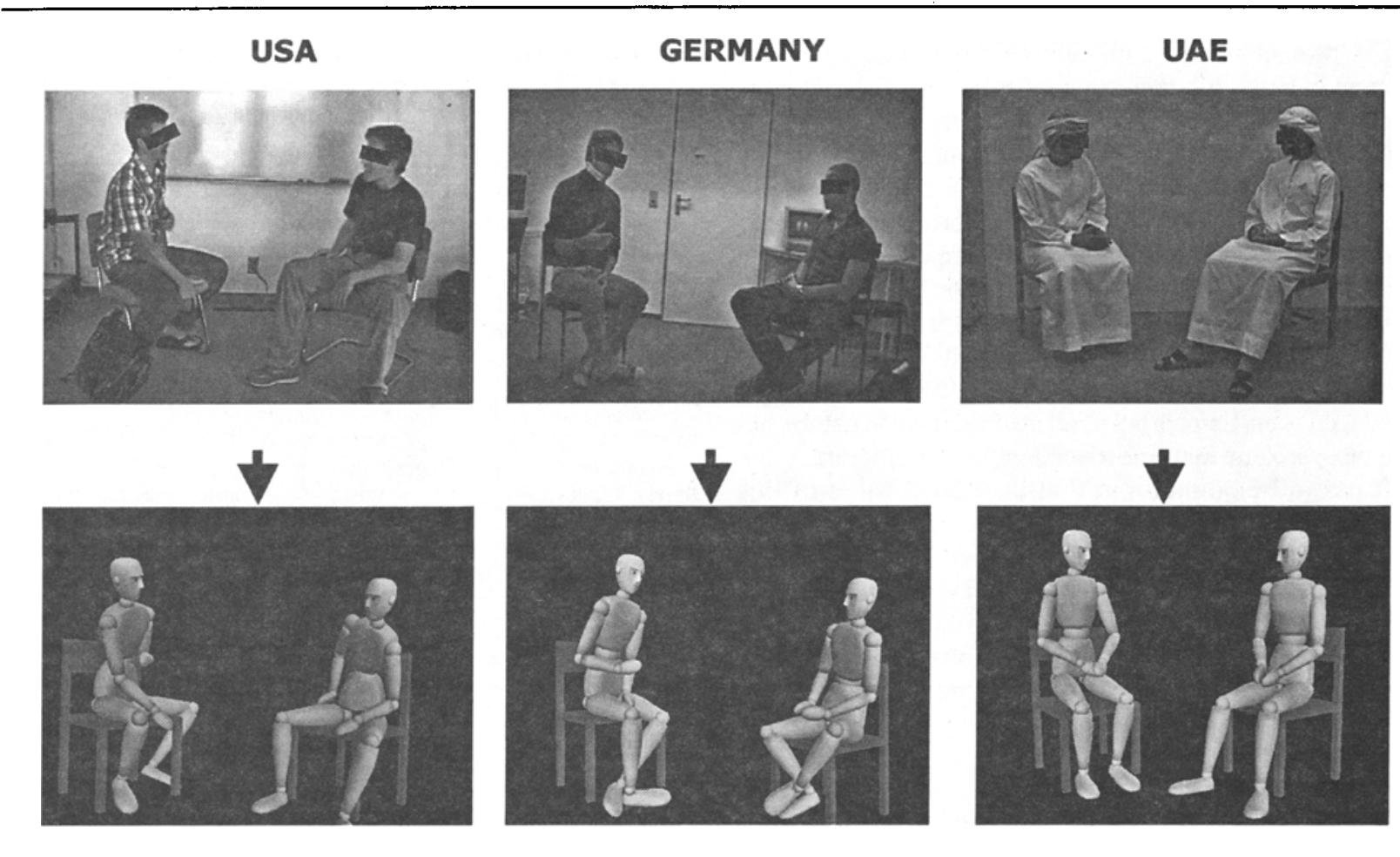

Figure 3. Snapshots from original videos for the United States, Germany, and the United Arab Emirates (UAE), respectively, with the corresponding screen shots from computer animations (for a description, see the text).

3-D animation sequences using standardized computer characters. These characters can be chosen to mask particularities of physical appearance that might hint at the ethnicity or cultural background of the actors on the screen. Exported AVI files dan be copied and used as stimulus material in person perception studies on other computers or; after conversion, even on CD players. Figure 3 gives an example of the resulting stimulus materials, showing still pictures from our cross-cultural study. The upper row shows stills from the original videos, and the lower row depicts the corresponding stills from the animations based on the time series transcripts. This example hints at the coding accuracy and also shows how aspects of physical appearance that might influence person perception can be effectively masked by using standardized computer characters.

As mentioned above, the movement protocols used for these animations are editable, and thus allow for more than the mere "masked replay" of computer animations. Cultural differences in NVB as identified in structural analysis can thus also be targeted more directly in controlled simulation studies, introducing systematic variations of behavior into the data protocols and then using the resulting animations as stimuli for person perception experiments. This principle has been successfully applied in an experimental study by Krämer (2001) that examined the effects of various head and body postures, as well as of different levels of movement activity, on person perception. She used an early predecessor of the actual coding tool, which in those days required an enormous coding effort. The present software tool allows for easy interactive manipulation of specific nonverbal cues-such as postures, gestures, or body movements-as well as for the algorithmic modification of complete data sets. For this purpose, external programs can be used to modify the exported ASCII files using simple search and replace or more complex filter routines. Our software plug-in is designed to reimport the modified movement protocols into Motion Builder and to render the new animations immediately. This strategy (for example) permits us to eliminate or insert culture-specific patterns as identified in the structural analysis. The resulting computer animations can be shown to observers from different cultures to verify the influence of particular cues on person perception within and across the cultures under investigation.

\section{Concluding Remarks}

A unified approach to the description and evaluation of NVB has been suggested, and a novel computer-based research tool has been introduced. The presented tool makes use of state-of-the-art 3-D character animation software to facilitate movement transcription and experimental simulation of NVB. It should be noted that computerassisted movement transcription is still a time-consuming process. However, in contrast to earlier approaches, this novel method provides a series of new graphical as well as numerical coding interfaces that allow for more convenient and reliable data entry, as well as for an unbiased, high-resolution, and highly accurate notation covering all aspects of body motion. 
The present version still suffers from a few constraints, such as the lack of finger movements and-more importantly - the lack of facial activity. As mentioned above, earlier studies demonstrated the ample importance of movement behavior for impression formation, showing that the lack of facial activity did not affect the correlations between judgments based on videos and on computer animations. Nevertheless, the inclusion of facial activities will widen the scope of possible studies enormously-for example, by extending the research questions to the area of cultural differences in the production and reception of emotional displays. In fact, this constraint is not of an insurmountable nature, but will be overcome in the next versions of our program.

It has to be pointed out that the added value of this novel methodology derives from the application of an exhaustive, unbiased coding procedure. The descriptive nature and unique information richness of the data sets enable researchers to pursue different research strategies, ranging from exploratory analyses of the movement protocols, to culture-fair judgment studies using 3-D dummies, up to hypothesis-driven person perception experiments that use computer-animated stimuli. Since the data collection procedure is not biased by stereotypes or theoretical preassumptions, the data protocols can be reused for consecutive studies targeting different aspects of NVB and different groups of observers. Moreover, the influence of stereotypes can be more directly studied by systematically varying the appearance of the 3-D characters while showing the same behavior, a technique that has not been possible at all in traditional person perception research.

\section{Availability}

The software application described here is realized as a plug-in and requires a valid license for Alias Motion Builder 7.5. The plug-in is available as a DLL for free on e-mail request to the first author.

\section{AUTHOR NOTE}

The methodological developments reported here have benefited from the financial support of the German Science Foundation (Grant DFG SFB/FK 427) and of the European Commission, within the project BIOSEC (Grant FP 5-IST). We gratefully acknowledge technical support by Takomat Cologne. Correspondence relating to this article may be sent to G. Bente, Department of Psychology, University of Cologne, BernhardFeilchenfeldstrasse 11, 50969 Cologne, Germany (e-mail: bente@uni -koeln.de).

\section{REFERENCES}

Argyle, M., Salter, V., Nicholson, H., Williams, M., \& Burgess, P. (1970). The communication of inferior and superior attitudes by verbal and non-verbal signals. British Journal of Social \& Clinical Psychology, 9, 222-231.

BENTE, G. (1989). Facilities for the graphical computer simulation of head and body movements. Behavior Research Methods, Instruments, \& Computers, 21, 455-462.

BENTE, G. (2002). Entschlüsselung einer ungewissen Botschaft: Zur Transkription und Analyse nonverbaler Kommunikationsprozesse. In L. Jäger \& G. Stanitzek (Eds.), Transkribieren: Medien/Lektüre. München: Wilhelm Fink.

Bente, G., D'Alessandro, M., FÜrTJes, M., \& KRÄmer, N. (1997).
Formale Angebotsweisen und interpersonelle Kommunikationsmuster im Affekt-Talk. In G. Bente \& B. Fromm (Eds.), Affektfernsehen: Motive, Angebotsweisen und Wirkungen (pp. 186-242). Opladen: Leske \& Budrich.

Bente, G., DonaGhy, W. C., \& SuWElack, D. (1998). Sex differences in body movement and visual attention: An integrated analysis of movement and gaze in mixed-sex dyads. Journal of Nonverbal Behavior, 22, 31-58.

Bente, G., Feist, A., \& Elder, S. (1996). Person perception effects of computer simulated male and female head movement. Journal of Nonverbal Behavior, 20, 213-228.

BENTE, G., \& KRÄMER, N. C. (2004). Inhaltsanalyse medialer Angebote. In R. Mangold, P. Vorderer, \& G. Bente (Eds.), Lehrbuch Medienpsychologie (pp. 201-228). Göttingen: Hogrefe.

Bente, G., Krämer, N. C., \& Petersen, A. (Eds.) (2002). Virtuelle Realitäten. Göttingen: Hogrefe.

Bente, G., KRÄmer, N. C., Petersen, A., \& De Ruiter, J. P. (2001). Computer animated movement and person perception: Methodological advances in nonverbal behavior research. Journal of Nonverbal Behavior, 25, 151-166.

Bente, G., Krämer, N. C., Trogemann, G., Piesk, J., \& Fischer, O. (2001). Conversing with electronic devices: An integrated approach towards the generation and evaluation of nonverbal behavior in face-to-face like interface agents I. In A. Heuer \& T. Kirste (Eds.), Intelligent interactive assistance and mobile multimedia computing (pp. 67-76). Rostock: Neuer Hochschulschriftenverlag.

BOAS, F., \& EFRON, D. F. (1936). A comparative investigation of gestural behavior patterns in "racial" groups living under different as well as similar environmental conditions. Psychological Bulletin, 33, 760.

Buck, R., Losow, J. I., MurPhy, M. M., \& Costanzo, P. (1992). Social facilitation and inhibition of facial expression and communication. Journal of Personality \& Social Psychology, 63, 962-968.

BURGOON, J. K. (1994). Nonverbal signals. In M. L. Knapp \& G. R. Miller (Eds.), Handbook of interpersonal communication (2nd ed., pp. 229 285). Thousand Oaks, CA: Sage.

Burgoon, J. K., Buller, D. B., \& WoOdall, W. G. (1996). Norverbal communication: The unspoken dialogue (2nd ed.). New York: McGraw-Hill.

Chen, M., \& BARGH, J. A. (1997). Nonconscious behavioral confirmation processes: The self-fulfilling consequences of automatic stereotype activation. Journal of Experimental Social Psychology, 33, 541-560.

DePaulo, B. M., \& Friedman, H. S. (1998). Nonverbal communication. In D. T. Gilbert, S. T. Fiske, \& G. Lindzey (Eds.), The handbook of social psychology (4th ed, Vol. 2, pp. 3-40). Boston: McGrawHill.

Dimberg, U. (1997). Psychophysiological reactions to facial expressions. In U. Segerstrale \& P. Molnár (Eds.), Nonverbal communication: Where nature meets culture (pp. 27-60). Mahwah, NJ: Erlbaum. DONAGHY, W. C. (1989). Nonverbal communication measurement. In P. Emmert \& L. Barker (Eds.), Measurement of communication behavior. White Plains, NY: Longman.

DOVIDIO, J. F., KAWAKAMI, K., \& GAERTNER, S. L. (2002). Implicit and explicit prejudice and interracial interaction. Journal of Personality \& Social Psychology, 82, 62-68.

DuNCAN, B. L. (1976). Differential social perception and attribution of intergroup violence-Testing lower limits of stereotyping of blacks. Journal of Personality \& Social Psychology, 34, 590-598.

EFron, D. (1941). Gesture and envinonment. Oxford: King's Crown Press.

Ekman, P. (1972). Universals and cultural differences in facial expressions of emotion. Paper presented at the Nebraska Symposium on Motivation, Lincoln, NE.

EKMAN, P. (1976). Movements with precise meanings. Journal of Communication, 26, 14-26.

EKMAN, P., \& FrIesEN, W. V. (1978). The facial action coding system. Palo Alto, CA: Consulting Psychologists Press.

Ekman, P., Friesen, W. V., O'Sullivan, M., Chan, A., DiacoyanNITARLATZIS, I., HEIDER, K., ET AL. (1987). Universals and culturaldifferences in the judgments of facial expressions of emotion. Journal of Personality \& Social Psychology, 53, 712-717. 
Frey, S. (1999). Die Macht des Bildes: Der Einfluss der nonverbalen Kommunikation auf Kultur und Politik. Bern: Huber.

Frey, S., Hirsbrunner, H. P., Florin, A., Daw, W., \& Crawford, R. (1983). A unified approach to the investigation of nonverbal and verbal behavior in communication research. In W. Doise \& S. Moscovici (Eds.), Current issues in European social psychology (pp. 143-199). Cambridge: Cambridge University Press.

Frey, S., HirsbrunNer, H. P., POOL, J., \& DaW, W. (1981). Das Berner System zur Untersuchung nonverbaler Interaktion: I. Die Erhebung des Rohdatenprotokolls. In P. Winkler (Ed.), Methoden der Analyse von face-to-face Situationen (pp. 203-237). Stuttgart: Metzler.

FREY, S., \& POOL, J. (1976). A new approach to the analysis of visible behavior. Bern: University of Bern.

FrIesen, W. V., EKMAN, P., \& WALlbotT, H. (1979). Measuring hand movements. Journal of Nonverbal Behavior, 4, 97-112.

Grammer, K., Honda, R., Juette, A., \& SchmitT, A. (1999). Fuzziness of nonverbal courtship communication: Unblurred by motion energy detection. Journal of Personality \& Social Psychology, 77, 487-508.

HAL.L, E. T. (1976). Beyond culture. Garden City, NY: Anchor.

HALL, E. T., \& HALL, M. R. (1990). Understanding cultural differences. Yarmouth, ME: Intercultural Press.

HOFSTEDE, G. (1980). Culture's consequences: International diffenences in work-related values. Newbury Park, CA: Sage.

KITAO, K., \& KITAO, S. K. (1989). Intercultural communication: Between Japan and the United States. Tokyo: Eichosha.

KOWNER, R., \& WISEMAN, R. (2003). Culture and status-related behavior: Japanese and American perceptions of interaction in asymmetric dyads. Cross-Cultural Research, 37, 178-210.

KRÄMER, N. C. (2001). Bewegende Bewegung: Sozio-emotionale Wirkungen nonverbalen Verhaltens und deren experimentelle Untersuchung mittels Computeranimation. Lengerich: Pabst.

KRUMHUBER, E., \& KapPas, A. (2005). Moving smiles: The role of dynamic components for the perception of the genuineness of smiles. Journal of Nomverbal Behavior, 29, 3-24.
KuDOH, T., \& MATsumoto, D. (1985). Cross-cultural examination of the semantic dimensions of body postures. Journal of Personality \& Social Psychology, 48, 1440-1446.

LeE, M. E., Matsumoto, D., Kobayashi, M., Krupp, D., \& Maniatis, E. F. (1992). Cultural influences on nonverbal behavior in applied settings. In R. S. Feldman (Ed.), Applications of nonverbal behavional theories and reseanch (pp. 239-261). Hillsdale, NJ: Erlbaum.

Lewis, R. J., Derlega, V. J., Shankar, A., Cochard, E., \& Finkel, L. (1997). Nonverbal correlates of confederates' touch: Confounds in touch research. Journal of Social Behavior \& Personality, 12, 821-830.

Matsumoto, D. (2006). Culture and nonverbal behavior. In V. Manusov \& M. Patterson (Eds.), Handbook of nonverbal communication (pp. 219-235). Thousand Oaks, CA: Sage.

Matsumoto, D., \& KUDOH, T. (1987). Cultural similarities and differences in the semantic dimensions of body postures. Journal of Nonverbal Behavior, 11, 166-179.

Mehrabian, A., \& FerRIs, S. R. (1967). Inference of attitudes from non-verbal communication in two channels. Journal of Consulting Psychology, 31, 248-252.

MEhrabian, A., \& WiENER, M. (1967). Decoding of inconsistent communication. Journal of Personality \& Social Psychology, 6, 109-1 14.

Newcombe, M. J., \& AshKaNASY, N. M. (2002). The role of affect and affective congruence in perceptions of leaders: An experimental study. Leadership Quarterly, 13, 601-614.

SUWELACK, D., \& BENTE, G. (1995). Zur Kommunikationskultur der Geschlechter-Nonverbale Korrelate der Beziehungsregulation in gegengeschlechtlichen Dyaden. Zeitschrift für Individualpsychologie, 20, 133-146.

(Manuscript received April 24, 2007; revision accepted for publication July 17, 2007.) 